\title{
What is the European Society of Thoracic Surgeons (ESTS) Uniportal VATS Interest Group (UVIG)?
}

The Uniportal VATS Interest Group (UVIG) is a Working Group of the European Society of Thoracic Surgeons (ESTS). The purpose of UVIG is to create a community of Thoracic Surgeons inside the ESTS with a specific interest in Uniportal VATS (UniVATS) as the preferred approach to intrathoracic conditions amenable to surgical diagnosis and treatment. In these years, UniVATS has become a significant part of major lung resections (especially in some areas of the World, as in Asia) and has revolutionised the way thoracic surgeons treat intrathoracic conditions. Up to now, the papers published in PubMed about UniVATS were more than 600. Nevertheless, we have no guidelines or recommendations. Therefore, despite a considerable number of articles, there are still many doubts, and controversies and UVIG might be an ideal place where ideas for new projects and initiatives spring up.

UVIG works in close collaboration with other relevant ESTS working groups (such as robotic group, pleural diseases or and minimally invasive working groups) and plans joint projects and activities. The main the activities of UVIG will include educational events on UniVATS and the creation of a database fully compatible with the existing ESTS database to facilitate the introduction of regulatory criteria to define the features of UniVATS into details and understand its distribution among the thoracic surgical community. Once a year, concurrently with the ESTS Annual Meeting, there is an UVIG business meeting.

The papers, which are collected in this thematic issue of the Journal of Thoracic Diseases, aims to present and to publish the topics for devising recommendations on Uniportal VATS. Through this issue, we hope to update the international literature and share different international experiences.

\section{Acknowledgments}

None.

\section{Footnote}

Conflicts of Interest: The authors have no conflicts of interest to declare.

Ethical Statement: The authors are accountable for all aspects of the work in ensuring that questions related to the accuracy or integrity of any part of the work are appropriately investigated and resolved. 


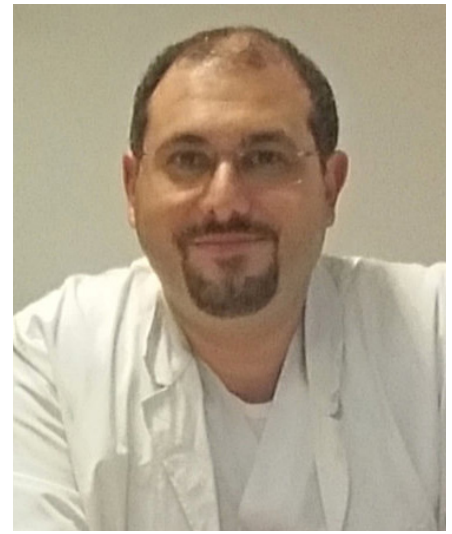

Luca Bertolaccini

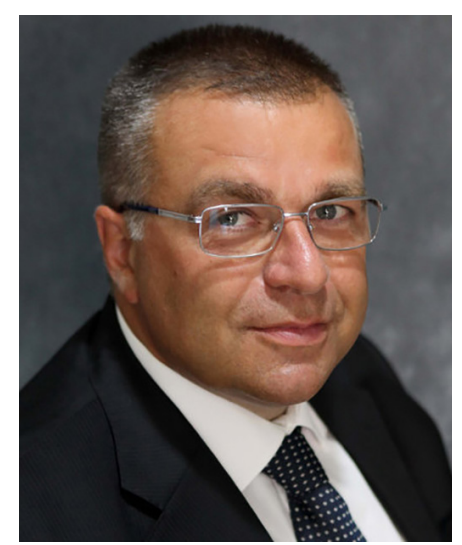

Gaetano Rocco

Luca Bertolaccini, MD, PhD, FCCP

Division of Thoracic Surgery, IEO, European Institute of Oncology IRCCS, Milan, Italy.

(Email: luca.bertolaccini@gmail.com)

Gaetano Rocco, MD, FRCSEd, FEBTS, FCCP

Department of Surgery, Memorial Sloan Kettering Cancer Center, New York, NY, USA.

(Email: gaetanorocco60@gmail.com) doi: $10.21037 /$ jtd.2019.06.55

View this article at: http://dx.doi.org/10.21037/jtd.2019.06.55

Cite this article as: Bertolaccini L, Rocco G. What is the European Society of Thoracic Surgeons (ESTS) Uniportal VATS Interest Group (UVIG)? J Thorac Dis 2019;11(Suppl 16):S2048-S2049. doi: 10.21037/jtd.2019.06.55 